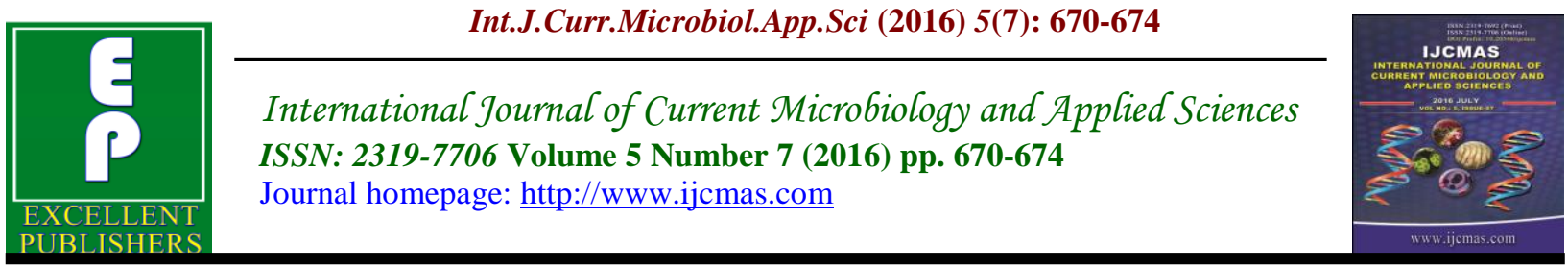

Original Research Article

http://dx.doi.org/10.20546/ijcmas.2016.507.076

\title{
A Study on Common Etiologies of Acute Febrile Illness Detectable by Microbiological Tests in a Tertiary Care Hospital
}

\author{
R. Vidhya Rani*, T. Sundararajan, S. Rajesh and T. Jeyamurugan \\ Department of Microbiology, Govt Mohan Kumaramangalam Medical College, \\ Salem Tamilnadu-636030, India \\ *Corresponding author
}

Keywords

Acute Febrile

Illness,

Primary Dengue

infection,

Thrombocytopenia and leucopenia.

Article Info

Accepted:

20 June 2016

Available Online:

10 July 2016

\section{A B S T R A C T}

Acute febrile illness is a common cause of patients seeking health care settings posing a diagnostic and therapeutic challenge to the health care workers. The aim of the study was to identify the common etiologies of acute febrile illness which are detectable by employing microbiological tests with correlation of laboratory parameters. This is a retrospective study involving 200 cases of acute febrile illness in a tertiary care hospital, Salem from December 2015-May 2016. Blood samples were tested for Dengue, Malaria, Typhoid, Leptospirosis, Rickettsial infections and complete blood count analysis. Males and persons with age group of 20-40 were commonly affected. The commonest cause of acute febrile illness was Dengue in 54 cases (27\%) with Primary Dengue infection in 45 cases (83\%) and Secondary dengue infection in 9 cases $(17 \%)$. Most cases of Dengue were reported in monsoon and post monsoon period. Other causes of acute febrile illness detected were Typhoid in 6cases (4\%), Malaria in 4cases(2\%) and Rickettsial infections in 2cases (1\%). Thrombocytopenia and leucopenia were marked in Dengue cases. Most Typhoid cases had leucopenia and Malaria cases had thrombocytopenia. Confirmatory diagnostic tests along with clinical skills and a good knowledge on epidemiology of febrile illness is essential in the management of such infections. Vector control measures along with public awareness on preventive measures needs to be strengthened.

\section{Introduction}

"Acute febrile illness", or "acute fever" or "short febrile illness" is traditionally defined as any illness associated with fever of two weeks or shorter in duration, rapid in onset, caused by diverse pathogens. The clinical signs and symptoms of most of these infections are very similar and the correct diagnosis is only possible by using pathogen specific diagnostic tests (Premaratna, 2013).
Several studies have documented the etiology of acute febrile illness in tropical countries like India as Dengue, Malaria, Typhoid fever, Leptospirosis, Rickettsial infection (Abraham et al., 2015). Climate variation, over population and urbanization may all contribute to the emergence and reemergence of infections in tropical regions like Tamilnadu (Singh et al., 2012). 
A retrospective study was done in a tertiary care hospital, Salem in Tamilnadu to identify the different etiologies of acute febrile illness caused by common infectious agents prevailing in the tropical countries with the aid of microbiological tests and laboratory parameters.

\section{Materials and Methods}

This is retrospective study done for six months involving 200 patients who had presented with acute febrile illness to a tertiary care hospital in Salem, Tamilnadu from December 2015-May 2016.

Specimen included 1) serum sample for performing microbiological tests such as Dengue, Typhoid, Leptospirosis, Rickettsial infections. 2) EDTA blood sample for Quantitative Buffy Coat analysis and complete blood count analysis.

\section{Methodology}

\section{Dengue}

Detection of NS1 Antigen done using Panbio Dengue early ELISA.

Detection of IgM and IgG done by Panbio IgM and IgG capture ELISA.

\section{Malaria}

Detection of Malarial parasites done by Quantitative Buffy Coat analysis using Parascan Malaria testing kit from Avantor.

\section{Typhoid}

Detection of Salmonella antibodies done by slide and tube agglutination method using Tydal kit from Tulip diagnostics.

\section{Rickettsial infection}

Serological diagnosis done by Heterophile agglutination test using Febrile Antigen Kit from Tulip diagnostics.

\section{Leptospirosis}

Detection of $\operatorname{IgM}$ antibodies done by Immunochromatography using Leptochek from Tulip diagnostics.

Complete blood count analysis done by ABX Three part Haematology analyzer from HORIBA

\section{Results and Discussion}

Early diagnosis of acute febrile illness in the developing tropical countries is the need of the hour. As many clinical features are overlapping and non specific, all these conditions present diagnostic challenges (Dhingra et al., 2011).

In this study a total of 200 cases who presented with acute febrile illness for a period of six months were evaluated. Microbiological tests with good sensitivity and specificity targeting the common causative agents of acute febrile illness in tropical countries like Dengue, Typhoid, Malaria, Leptospirosis, Rickettsial infections were performed for all these patients. Cases of acute fever in other tropical regions have been identified and documented in several studies ( Kulkarni et al., 2010).

In our study, the commonest cause of acute febrile illness was Dengue in 54 cases $(27 \%)$. Other causes detected were Typhoid in 6cases (3\%), Malaria in 4cases $(2 \%)$ and Rickettsial infections in 2cases (1\%). Yogeesha et al., (2014) in had a similar observation in their study in South India on acute febrile illness where most patients had Dengue, Malaria, Leptospirosis, typhoid. Neelu sree et al., (2015) had also reported Dengue, Malaria, Scrub typhus and Leptospirosis in their study. 
Table.1 Etiologies of Acute febrile illness

\begin{tabular}{|l|l|l|l|l|l|}
\hline Total cases & Dengue & Typhoid & Malaria & $\begin{array}{l}\text { Rickettsial } \\
\text { infections }\end{array}$ & Others* \\
\hline 200 & 54 & 6 & 4 & 2 & 134 \\
\hline
\end{tabular}

*-Others include viral infections, bacterial infections and undetectable causes

Table.2 Gender wise distribution of cases

\begin{tabular}{|l|l|l|l|l|l|l|}
\hline Gender & Dengue & Typhoid & Malaria & $\begin{array}{l}\text { Rickettsial } \\
\text { infections }\end{array}$ & Others & Total \\
\hline Male & 29 & 3 & 3 & 2 & 81 & 118 \\
\hline Female & 25 & 3 & 1 & 0 & 53 & 82 \\
\hline
\end{tabular}

Table.3 Age wise distribution of cases

\begin{tabular}{|l|l|l|l|l|l|l|}
\hline Age & Dengue & Typhoid & Malaria & $\begin{array}{l}\text { Rickettsial } \\
\text { infections }\end{array}$ & Others & Total \\
\hline $0-20$ & 17 & - & - & - & 20 & 37 \\
\hline $20-40$ & 21 & 3 & 2 & 2 & 52 & 80 \\
\hline $40-60$ & 12 & 3 & 2 & - & 41 & 58 \\
\hline $60-80$ & 4 & - & - & - & 21 & 25 \\
\hline
\end{tabular}

Table.4 Month wise distribution of acute febrile cases

\begin{tabular}{|l|l|l|l|l|l|}
\hline Month & Dengue & Typhoid & Malaria & $\begin{array}{l}\text { Rickettsial } \\
\text { infections }\end{array}$ & Others \\
\hline December & 24 & 2 & 2 & 1 & 36 \\
\hline January & 11 & 2 & 1 & - & 21 \\
\hline February & 9 & 1 & 1 & 1 & 15 \\
\hline March & 4 & 1 & - & - & 17 \\
\hline April & 3 & - & - & - & 21 \\
\hline May & 2 & - & - & - & 28 \\
\hline
\end{tabular}

Table.5 Thrombocytopenia and leucopenia in cases with acute febrile illness

\begin{tabular}{|l|l|l|l|}
\hline Lab parameters & Dengue & Typhoid & Malaria \\
\hline Thrombocytopenia & 40 & - & 3 \\
\hline Leucopenia & 34 & 3 & 1 \\
\hline
\end{tabular}

Table.6 Primary and secondary Dengue infection

\begin{tabular}{|l|l|l|}
\hline Dengue cases & Primary dengue infection & Secondary dengue infection \\
\hline 54 & $45(83 \%)$ & $9(17 \%)$ \\
\hline
\end{tabular}




\section{Etiologies of Acute febrile illness}

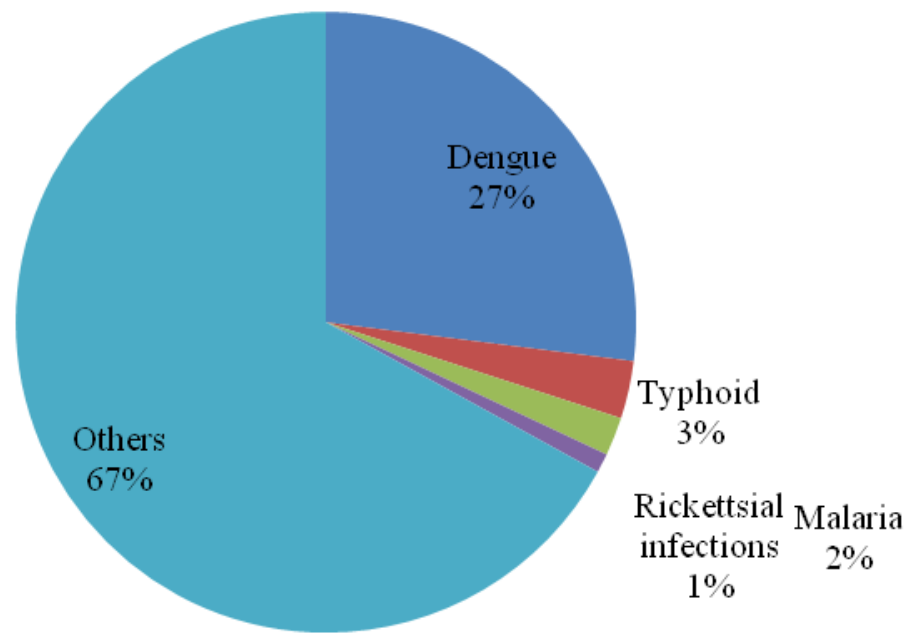

Other cases of acute febrile illness of our study include viral infections, bacterial infections and undectable causes. Males were commonly affected with total of 118 cases of acute febrile illness of which detectable cases were 37 as compared to females with 29 detectable cases. Patients in age group 20-40 were predominantly affected. Abraham et al., 2015 had documented men preponderance with most of the patients in the productive phase of life.

In this study many cases were reported in December and January which is the monsoon and post monsoon period in our place in Tamilnadu. Jhansi Charles et al., (2015) and Priyadarshini Shanmugam et al., (2016) had shown peak incidence of cases during rainy and post monsoon seasons in their study. Since large number of cases occur during monsoon period, preventive measures have to initiated promptly like control of mosquitoes, rodents .The general public have to be educated and awareness should be created on good hygienic practices, vector control measures and protective measures.

In our study thrombocytopenia and leucopenia were more marked in Dengue cases with 40 cases of thrombocytopenia and 34 cases of leucopenia. Most cases of Typhoid had leucopenia and Malaria had thrombocytopenia. These findings on laboratory parameters correlated with the study done by Yogeesha et al., (2014)

In this study among Dengue cases, Primary Dengue infections accounted for about $83 \%$ and Secondary dengue infection for $17 \%$. Patients with secondary dengue infection are more prone to develop complications like Dengue haemorrhagic fever and Dengue shock syndrome and those patients have to be managed effectively to prevent the mortality.

In conclusion, although Dengue being the commonest cause of acute febrile illness, other different causes prevailing in tropical countries should be suspected and correct 
diagnosis using is crucial to prevent the delay in starting appropriate therapy. Confirmatory diagnostic tests with good correlation of clinical findings, relevant laboratory parameters and epidemiology of disease is essential to prevent complications and to reduce morbidity and mortality in patients with acute febrile illness. Vector control measures and public awareness regarding surveillance and preventive measures have to be strengthened to reduce the disease burden.

\section{References}

Abraham, M., Ittyachen, Reshmi Ramachandran. 2015. Study of acute febrile illness: A 10-year descriptive study and a proposed algorithm from a tertiary care referral hospital in rural Kerala in southern India. Tropical Doctor, Vol.45(2): 114-117.

Dhingra, B., Mishra, D. 2011. Early diagnosis of febrile illness: the need of the hour. Indian Pediatr., 48: 845-849.

Jhansi Charles, Ramesh, A., Anand Janagond, Rajendran, T., Thilakavathy, P., Vithiya, G. 2015. Studu of Prevalence of Dengue infection in aRurally situated Tericare care Medical College Hospital at Madurai, Tamilnadu. J. Dental and Med. Sci., Volume 14, Issue 10. Ver.V.
Kulkarni, R.D., Batra, H.V., Tuteja, U., Shukla, J., Patil, S.A., Kulkarni, V.A., et al. 2010. Investgation into an outbreak of acute febrile illness in Sangali district of Maharasthra state. 2010.India. Int. J. Clin. Pract., 64: 9596.

Neelu Sree, P., Premkumar, L. 2015. A pilot styudy on Acute undifferentiated fever using certain rapid microbiological and virology tests. Int. J. Pharm. Bio. Sci., 6(4):716-723.

Premaratna, R. 2013. Dealing with Acute Febrile Illness in the Resource Poor Tropics. Trop. Med. Surg., 1: 101.

Priyadarshini Shanmugam, Nirupa Soundararajan, Vidhya Ravi, Preethi Venkatesan. 2016. A study on the prevalence of dengue fever in Kelambakkam in comparison to an earlier study. Indian J. Microbiol. Res., 3(2): 102-106.

Singh, P.K., Dhiman, R.C. 2012. Climate change and human health. J. Vector Borne Dis., 49: 55-60.

Yogeesha, K.S., Sreejith M.G, Raghavendra Bakki Sannegowda, Manjunath, J. 2014. Clinical and Laboratory parameters differentiating Dengue from other causes of Acute Febrile illnesses in a Teritary care centre in South India. Int. J. Recent Trends in Sci. Technol., Volume 10, Issue 2.

\section{How to cite this article:}

Vidhya Rani, R., T. Sundararajan, S. Rajesh and Jeyamurugan, T. 2016. A Study on Common Etiologies of Acute Febrile Illness Detectable by Microbiological Tests in a Tertiary Care Hospital. Int.J.Curr.Microbiol.App.Sci. 5(7): 670-674. doi: http://dx.doi.org/10.20546/ijcmas.2016.507.076 